\title{
Tables of Complete Elliptic Integrals
}

\section{J. M. Hammersley ${ }^{2}$}

The complete elliptic integrals

$$
\begin{aligned}
K=\int_{0}^{\pi / 2}\left(1-k^{2} \sin ^{2} \phi\right)^{-\frac{1}{2}} d \phi, \\
E=\int_{0}^{\pi / 2}\left(1-k^{2} \sin ^{2} \phi\right)^{\frac{1}{2}} d \phi
\end{aligned}
$$

and the associated function

$$
M=\frac{\pi}{2 K}
$$

have been tabulated extensively ${ }^{34}$ with $k^{2}$ and $k$ and

\footnotetext{
1 This paper was prepared under a National Bureau of Standards contract with American University.

Oxford University and American Iniversity.

3 A. Fletcher, J. C. P. Miller, and L. Rosenhead, An index of mathematical

\begin{tabular}{|c|c|c|c|}
\hline $1 / k$ & $K$ & $E$ & $M$ \\
\hline $\begin{array}{l}\text { 1. } 00 \\
\text { 1. } 01 \\
\text { 1. } 02 \\
\text { 1. } 03 \\
\text { 1. } 04\end{array}$ & \begin{tabular}{lc}
\multicolumn{1}{c}{$\infty$} \\
3. 36145 & 8120 \\
3. 03047 & 9311 \\
2. 84195 & 7373 \\
2. 71141 & 0062
\end{tabular} & 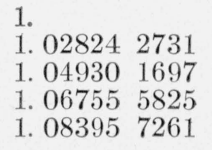 & $\begin{array}{ll}0 . & \\
0.46729 & 61170 \\
0.51833 & 26351 \\
0.55271 & 63573 \\
0.57932 & 82058\end{array}$ \\
\hline $\begin{array}{l}\text { 1. } 05 \\
\text { 1. } 06 \\
\text { 1. } 07 \\
\text { 1. } 08 \\
\text { 1. } 09\end{array}$ & $\begin{array}{ll}\text { 2. } 61243 & 9112 \\
\text { 2. } 53331 & 9380 \\
\text { 2. } 46781 & 4067 \\
\text { 2. } 41221 & 1023 \\
\text { 2. } 36412 & 2792\end{array}$ & $\begin{array}{l}\text { 1. } 098966268 \\
\text { 1. } 112858448 \\
\text { 1. } 125818774 \\
\text { 1. } 137980652 \\
\text { 1. } 149445208\end{array}$ & $\begin{array}{ll}\text { 0. } 60127 & 57656 \\
\text { 0. } 62005 & 45970 \\
0.63651 & 32398 \\
0.65118 & 52868 \\
0.66443 & 09393\end{array}$ \\
\hline $\begin{array}{l}\text { 1. } 10 \\
\text { 1. } 11 \\
\text { 1. } 12 \\
\text { 1. } 13 \\
\text { 1. } 14\end{array}$ & $\begin{array}{ll}\text { 2. } 32192 & 4037 \\
\text { 2. } 28445 & 8951 \\
\text { 2. } 25087 & 6725 \\
\text { 2. } 22053 & 3243 \\
\text { 2. } 19292 & 9400\end{array}$ & $\begin{array}{ll}\text { 1. } 16029 & 1946 \\
\text { 1. } 17058 & 5138 \\
\text { 1. } 18037 & 7893 \\
\text { 1. } 18971 & 4883 \\
\text { 1. } 19863 & 4233\end{array}$ & $\begin{array}{ll}\text { 0. } 67650 & 63378 \\
\text { 0. } 68760 & 10297 \\
\text { o. } 69785 & 97760 \\
\text { 0. } 70739 & 59970 \\
0.71630 & 04550\end{array}$ \\
\hline $\begin{array}{l}\text { 1. } 15 \\
\text { 1. } 16 \\
\text { 1. } 17 \\
\text { 1. } 18 \\
\text { 1. } 19\end{array}$ & $\begin{array}{ll}\text { 2. } 16767 & 0799 \\
\text { 2. } 14444 & 0528 \\
\text { 2. } 12298 & 0225 \\
\text { 2. } 10307 & 6584 \\
\text { 2. } 08455 & 1532\end{array}$ & 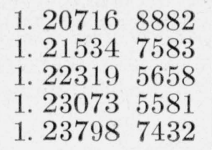 & $\begin{array}{ll}\text { 0. } 72464 & 70855 \\
0.73249 & 70342 \\
0.73990 & 15350 \\
0.74690 & 40066 \\
0.75354 & 16145\end{array}$ \\
\hline $\begin{array}{l}\text { 1. } 20 \\
\text { 1. } 21 \\
\text { 1. } 22 \\
\text { 1. } 23 \\
\text { 1. } 24\end{array}$ & $\begin{array}{ll}\text { 2. } 06725 & 4932 \\
\text { 2. } 05105 & 9081 \\
\text { 2. } 03585 & 4498 \\
\text { 2. } 02154 & 6642 \\
\text { 2. } 00805 & 3348\end{array}$ & 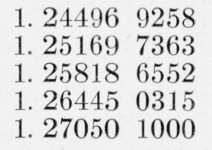 & $\begin{array}{ll}0.75984 & 64529 \\
0.76584 & 64553 \\
0.77156 & 61060 \\
\text { 0. } 77702 & 70023 \\
0.78224 & 83048\end{array}$ \\
\hline $\begin{array}{l}\text { 1. } 25 \\
\text { 1. } 26 \\
\text { 1. } 27 \\
\text { 1. } 28 \\
\text { 1. } 29\end{array}$ & 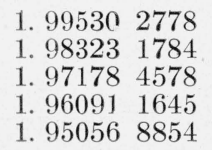 & 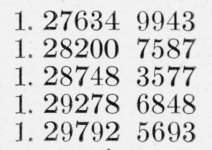 & $\begin{array}{ll}\text { 0. } 78724 & 71008 \\
0.79203 & 87014 \\
0.79663 & 68863 \\
0.80105 & 41070 \\
0.80530 & 16554\end{array}$ \\
\hline $\begin{array}{l}\text { 1. } 30 \\
\text { 1. } 32 \\
\text { 1. } 34 \\
\text { 1. } 36 \\
\text { 1. } 38\end{array}$ & 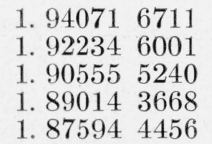 & $\begin{array}{ll}\text { 1. } 30290 & 7829 \\
\text { 1. } 31243 & 0289 \\
\text { 1. } 32140 & 6374 \\
\text { 1. } 32988 & 1897 \\
1.33789 & 7365\end{array}$ & $\begin{array}{ll}0.80938 & 98084 \\
0.81712 & 46622 \\
0.82432 & 47395 \\
0.83104 & 59960 \\
0.83733 & 62664\end{array}$ \\
\hline
\end{tabular}
tables (Scientific Computing Service, London, 1946)

4 A. Fletcher, Guide to tables of elliptic functions, MTAC 3, 229-281 (1948).
}

$\arcsin k$ as arguments. This replication of tables with a variety of closely related arguments is partly due to the difficulty of interpolating in the neighborhood of $k=1$. Legendre considered that interpolation was unsatisfactory in the range $1 \leq 1 / k \leq \sqrt{ } 2$. Recently the author needed tables of $K$ and $E$ with $1 / k$ as argument. This is quite a common need. For example, it arises in evaluating integrals of the form

$$
\int_{0}^{1} \frac{d x}{\sqrt{ }\left\{\left(1-x^{2}\right)\left(p^{2}-x^{2}\right)\right\}}, \quad p k=1 .
$$

The tables, given below, were computed by Gauss's arithmetic-geometric mean. The last significant figure is doubtful, but is retained in accordance with Fisher and Wishart. ${ }^{5}$

${ }^{5}$ R. A. Fisher and J. Wishart, On the distribution of the error of an interpolated value, and on the construction of tables, Proc. Cambridge Phil. Soc. 23, 912-921 (1927).

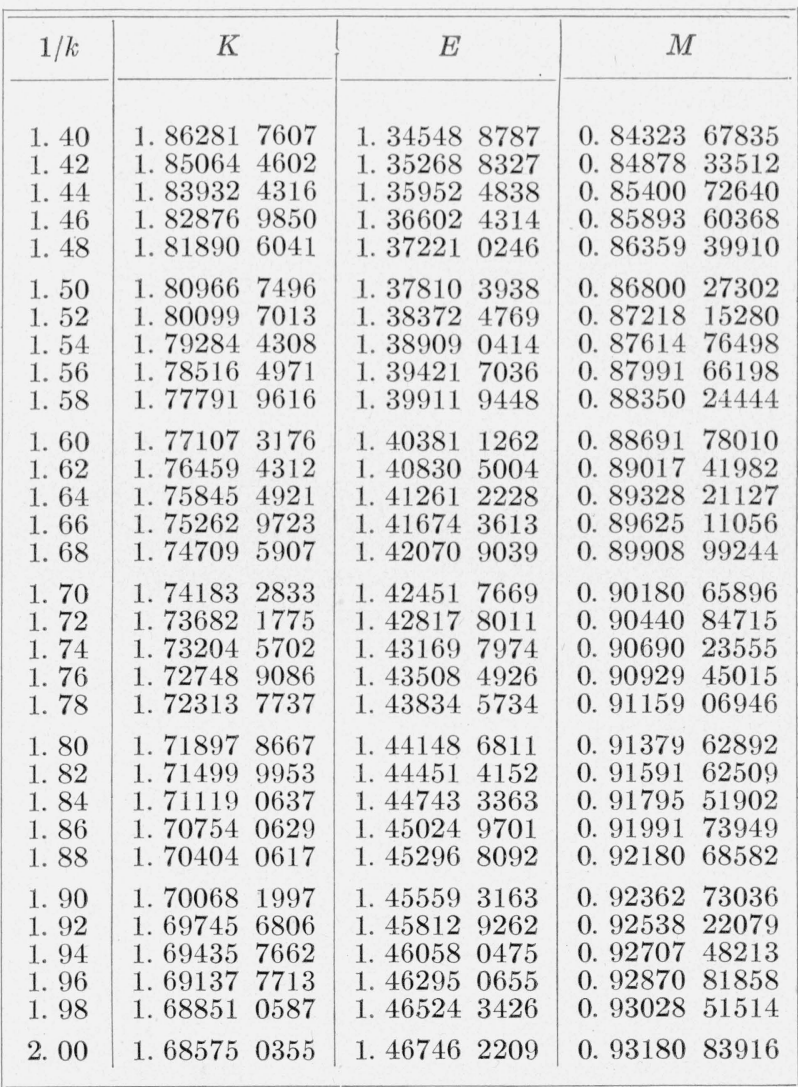

Washington, October 27, 1952. 